\title{
SUPERIMPRIMITIVE 2-GENERATOR FINITE GROUPS
}

\author{
by A. M. MACBEATH*
}

(Received 10th August 1985)

\section{Introduction}

In his thesis, A. A. Hussein Omar, motivated by the study of possible shapes of generic Dirichlet regions for a surface group, made a detailed study for $g=2,3$ of the groups generated by pairs $(\mu, \tau)$ of regular (i.e. fixed-point-free) permutations of order 2,3 respectively and of degree $n=6(2 g-1)$, such that $\mu \circ \tau$ is an $n$-cycle. He observed that, for $g=2,3$, precisely one pair generates what he calls a superimprimitive group, and raised the question whether such pairs exist for all $g$, and, if so, whether they are unique. Our main result is that they do always exist, but that, for large values of $g$, they are far from unique. (For details and some motivation for the notation, see $[4,5]$.)

We begin by constructing pairs $(\mu, \tau)$ of prime power degree, dropping the condition that the permutations should be regular. These pairs are very naturally described by graphs with edges of two colours. This powerful aid to intuition has been used with good effect by Stothers $[6,7]$ and Conder $[1,2]$ in a context very similar to ours. The technique seems to have originated in unpublished notes of Graham Higman. We then obtain regular permutations of degree $6(2 g-1)$ by taking tensor products of permutations of prime-power degree.

\section{Background}

In this section we recall some standard concepts and define superimprimitivity. A permutation of degree $n$ will, for us, be a group of bijections of the ring $\mathbb{Z} / n$ of residue classes $\bmod n$, the permutation $\sigma$ being defined once and for all by the equation

$$
\sigma(r)=r+1
$$

Occasionally we shall write $\sigma_{n}$, in case there is some doubt about the degree.

If $m, n$ are relatively prime integers, we identify $\mathbb{Z} / m n$ and the product $\mathbb{Z} / m \times \mathbb{Z} / n$ in the canonical way. If $\tau_{1}, \tau_{2}$ are two permutations of degrees $m, n$ respectively, the permutation $\tau_{1} \times \tau_{2}$ is defined by the formula

$$
\left(\tau_{1} \times \tau_{2}\right)(x, y)=\left(\tau_{1}(x), \tau_{2}(y)\right)
$$

In particular we have

$$
\sigma_{m n}=\sigma_{m} \times \sigma_{n}
$$

*This paper forms part of the Proceedings of the conference Groups-St Andrews 1985. 
Also if $\mu_{1}, \mu_{2}$ are two other permutations of degrees $m, n$ respectively then

$$
\left(\mu_{1} \times \mu_{2}\right) \circ\left(\tau_{1} \times \tau_{2}\right)=\left(\mu_{1} \circ \tau_{1}\right) \times\left(\mu_{2} \circ \tau_{2}\right) \text {. }
$$

If $\tau$ is a permutation of degree $n$ and $q$ is an equivalence relation on $\mathbb{Z} / n$ then $q$ and $\tau$ are said to be compatible ( $\tau$ is compatible with $q$ or $q$ is compatible with $\tau$ ) if

$$
\text { rqs implies } \tau(r) \mathfrak{q} \tau(s) \text {. }
$$

The set of all permutations compatible with $q$ is a group, and any group whose elements are all permutations compatible with $q$ is itself said to be compatible with $q$. If a compatible group is transitive, then all the equivalence classes have the same number of elements. In this case it is usual to call the equivalence classes blocks and the equivalence relation a block system. If $d$ is the number of blocks, and $d^{\prime}$ the number of elements in each block, then $n=d d^{\prime}$. If either $d=1$ or $d^{\prime}=1$, the block system is called trivial, since every group is plainly compatible. If there is no non-trivial block system compatible with a group $G$, then $G$ is primitive. Otherwise it is imprimitive.

A property completely opposite to primitivity has recently been introduced by Hussein [4]. $G$ is superimprimitive if, for each factorization $n=d d^{\prime}$, there is a block system compatible with $G$ consisting of $d$ blocks each with $d^{\prime}$ elements. Thus a group of prime degree is both primitive and superimprimitive. The regular representation of $G$ is superimprimitive if and only if $G$ satisfies the converse of Lagrange's Theorem. Also every transitive nilpotent permutation group is superimprimitive. These examples alone seem to indicate that superimprimitive groups are well worth further investigation. For the rest of this paper, however, we shall consider only a smaller family of permutation groups more directly relevant to Hussein's problem.

\section{Special superimprimitive groups}

Suppose that $d$ is a factor of $n$. The equivalence relation on $\mathbb{Z} / n$ of congruence modulo $d$ is called a special block system. A group of permutations of degree $n$ is called a special superimprimitive group if it is compatible with all the special block systems, that is for $d \mid n$ and for all $g \in G$,

$$
r \equiv s(\bmod d) \quad \text { implies } \quad g(r) \equiv g(s)(\bmod d) .
$$

The special block systems are plainly the only block systems compatible with the permutation $\sigma$, and we therefore have

Proposition 1. If $\sigma \in G$ then $G$ is superimprimitive if and only if it is a special superimprimitive group.

For each $n$, there is a unique maximal special superimprimitive group $S S(n)$ defined to be the set of all permutations of degree $n$ compatible with the special block systems. We now determine the structure of $S S(n)$. In the following theorem, the direct product $G \times H$ of two permutation groups of relatively prime degree $m, n$ respectively, is to be 
interpreted as the set of all permutations

$$
\{\sigma \times \tau \mid \sigma \in G, \tau \in H\}
$$

the product of permutations being defined as in (2.1) (the tensor product).

Theorem 1. If $(m, n)=1$, then $S S(m n)=S S(m) \times S S(n)$.

Corollary. If the prime factorization of $n$ is $n=p_{1}^{\alpha_{1}} p_{2}^{\alpha_{2}} \ldots p_{k}^{\alpha_{k}}$ then $\operatorname{SS}(n)=\operatorname{SS}\left(p_{1}^{\alpha_{1}}\right) \times$ $S S\left(p_{2}^{\alpha_{2}}\right) \times \cdots \times S S\left(p_{k}^{\alpha_{k}}\right)$.

Proof. If $g \in S S(m n)$, then $g$ induces a permutation $g_{1}$ of the residue classes $\bmod m$ and a permutation $g_{2}$ of the residue classes $\bmod n$. Thus $g$ is of the form $g_{1} \times g_{2}$. Since $g$ is compatible with congruence modulo every factor of $m$, so is $g_{1}$, and similarly $g_{2}$ is compatible with congruence modulo every factor of $n$. Thus $S S(m n)$ is contained in $S S(m) \times S S(n)$. The reverse inclusion is immediate.

The corollary of Theorem 1 shows that we need now only consider the case of primepower degree. Consider first the case of a finite set $\Omega$ of cardinality $n=d d^{\prime}$. A system of $d$ blocks each with $d^{\prime}$ elements can be regarded as a product decomposition

$$
\Omega=A \times B
$$

where $B$ has $d$ ' elements and the blocks are the "fibres" $\{a\} \times B$. A permutation $g$ acting on $\Omega$ is compatible with this block system if and only if the first coordinate of the $g$ image of $(a, b)$ depends only on the first coordinate $a$; that is, there is a uniquely defined permutation $g^{*}$ of $A$ such that

$$
g(a, b)=\left(g^{*}(a), h_{a}(b)\right)
$$

where, for each $a \in A, h_{a}$ is some permutation of $B$. The set of all permutations of the form (3.2) defines the wreath product (exactly as given, in particular, in Marshall Hall's book [3, p. 81]) so we see that the group of all permutations compatible with this single block system is the wreath product

$$
S_{d} \cdot w r S_{d}
$$

where, as usual $S_{k}$ denotes the symmetric group on $k$ elements. If in particular we take $d=d^{\prime}=p$, where $p$ is prime, we derive

$$
S S\left(p^{2}\right)=S_{p} w r S_{p}
$$

A straightforward extension of the argument to $n$ factors yields:

Theorem 2. $\operatorname{SS}\left(p^{n}\right)$ is the $n$-fold iterated wreath product with itself of the symmetric group $S_{p}$ 


\section{Permutation-pairs}

A pair $(\mu, \tau)$ of permutations of degree $n$ is called a $(2,3)_{n}$-pair if

$$
\mu^{2}=\tau^{3}=i d, \quad \mu \circ \tau=\sigma
$$

The $(2,3)_{n}$-pair is called superimprimitive if the group $\langle\mu, \tau\rangle$ which it generates is superimprimitive, so that, since $\sigma \in G$, by Proposition 1 it is then a special superimprimitive group. Thus $(\mu, \tau)$ is superimprimitive if and only if, for $d \mid n, x \equiv y(\bmod d)$ implies both $\mu(x) \equiv \mu(y)(\bmod d)$ and $\tau(x) \equiv \tau(y)(\bmod d)$.

The $(2,3)_{n}$-pair is called regular if $\mu$ and $\tau$ are both regular permutations. In that case $n$ must be an odd multiple of 6 , say $n=6(2 g-1)$. (See $[4,5]$.) In constructing regular $(2,3)_{n}$-pairs which are superimprimitive, the following theorem is an essential tool.

Theorem 3. If $(m, n)=1$, if $\left(\mu_{1}, \tau_{1}\right)$ is $a(2,3)_{m}$-pair and $\left(\mu_{2}, \tau_{2}\right)$ is $a(2,3)_{n}$-pair then

$$
\left(\mu_{1} \times \mu_{2}, \tau_{1} \times \tau_{2}\right)
$$

is a $(2,3)_{m n}$-pair. Moreover, if one of $\mu_{1}, \mu_{2}$ is regular and one of $\tau_{1}, \tau_{2}$ is regular then the product (4.1) is regular.

Proof. All the assertions, apart from the regularity at the end, follow from (2.2), (2.3). Now suppose that $\mu_{1} \times \mu_{2}$ is not regular, i.e., it has a fixed point $(x, y) \in \mathbb{Z} / m \times \mathbb{Z} / n$. Then $\mu_{1}(x)=x$ and $\mu_{2}(y)=y$ by $(2.1)$, so neither $\mu_{1}$ nor $\mu_{2}$ is regular. The regularity of $\tau_{1} \times \tau_{2}$ follows similarly.

Now, for a moment, consider permutations with domain a set $\Omega$ of $n$ elements, not initially given the ring structure $\mathbb{Z} / n$. A pair $(\mu, \tau)$ of permutations of $\Omega$ with $\mu^{2}=\tau^{3}=i d$ can be represented by a graph with vertex set $\Omega$ and with edges of two colours; undirected red edges joining a point of $\Omega$ to its $\mu$-image if it is not fixed by $\mu$; directed blue edges joining a point of $\Omega$ to its $\tau$-image, if it is not fixed by $\tau$. Points which are fixed by $\mu$ are not incident with any red edge, and points which are fixed by $\tau$ are not incident with any blue edge. Thus the graph is a union of red edges, no two of which have a vertex in common, and a set of blue oriented triangles, no two of which have a vertex in common. Conversely, such a graph always defines a pair of permutations $(\mu, \tau)$ with $\mu^{2}=\tau^{3}=i d$.

The pair $(\mu, \tau)$ can be regarded as a $(2,3)_{n}$-pair if $\mu \circ \tau$ is an $n$-cycle. For in this case we may write $\mu \circ \tau=\sigma$ and pick a vertex $v$ to be the zero-vertex. By associating $\sigma^{r}(v)$ with the residue-class $r(\bmod n)$, we identify $\Omega$ with $\mathbb{Z} / n$, and $(\mu, \tau)$ now is a $(2,3)_{n}$-pair.

We denote the graph described above with undirected red edges and directed blue edges by $\Gamma(\mu, \tau)$-where $\mu$ and $\tau$ are the permutations from which it is derived. There do not appear to be intuitively simple necessary and sufficient conditions on $\Gamma$ for $\mu \circ \tau$ to be an $n$-cycle. However, we shall derive a sufficient condition below which will be enough for our purpose. When there is no doubt about $\mu, \tau$, we shall simply write $\Gamma$ instead of $\Gamma(\mu, \tau)$. 
As a preliminary, we restrict attention to graphs $\Gamma(\mu, \tau)$ satisfying the following:

$$
\Gamma(\mu, \tau) \text { is connected. }
$$

No pair of vertices is joined by both a red edge and a blue edge.

These are both necessary for $\mu \circ \tau$ to be an $n$-cycle, since the first condition asserts that the group $\langle\mu, \tau\rangle$ is transitive, while the second asserts that $\mu \circ \tau$ has no fixed point.

(4.3) being satisfied, we can construct a graph $\Gamma_{1}$ from $\Gamma$ by collapsing all blue triangles to single points. All vertices will have valency 1,2 or $3 . \Gamma_{1}$ will have no loops by (4.3), and it will be connected by (4.2). A free vertex of $\Gamma_{1}$ can correspond either to a free vertex of $\Gamma$ or to a triangle in $\Gamma$; a vertex of valency 2 or 3 in $\Gamma_{1}$ must correspond to a triangle in $\Gamma$, since two red edges in $\Gamma$ cannot have a vertex in common. We shall refer to $\Gamma_{1}$ as the collapsed graph of the pair $(\mu, \tau)$.

Example. Let $\Gamma_{1}$ be the graph with two vertices and three distinct red edges joining them. Then $\Gamma$ must consist of two blue triangles with the vertices of one joined in some order to the vertices of the other. The choice of orientation gives two possibilities for $\Gamma$. In Fig. 1a we find $\mu \circ \tau=(123456)$, while in Fig. 1b we find $\mu \circ \tau=(12)(34)(56)$. Thus a decision whether $\mu \circ \tau$ is an $n$-cycle cannot be made, in general, from the study of $\Gamma_{1}$ alone. Unexpectedly, however, we have:
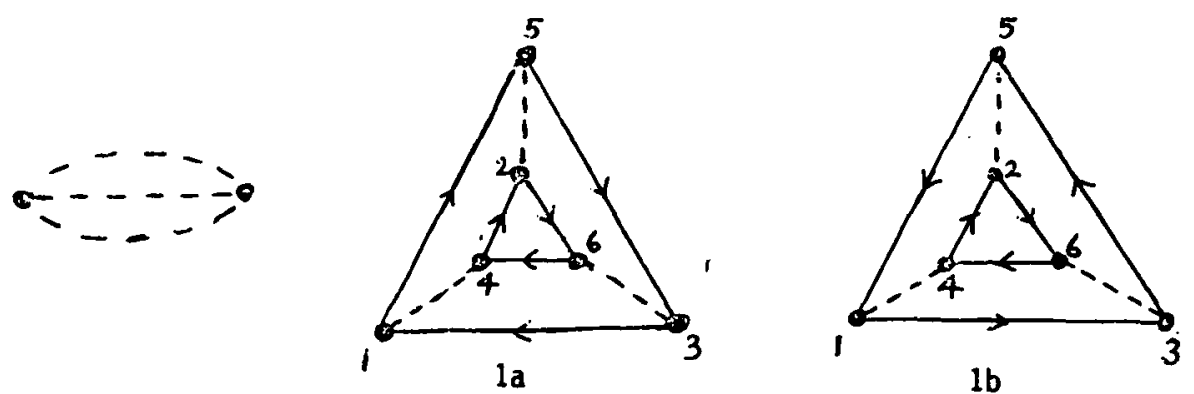

FIGURE 1 Two graphs of degree 6 with the same collapsed graph (on left). Red edges are broken, blue edges are solid.

Theorem 4. If $\Gamma_{1}$ is a tree, $\mu \circ \tau$ is an $n$-cycle.

Proof. By induction on the number $n$ of vertices of $\Gamma$. The small values $n=1,2,3,4$ yield one graph each satisfying (4.2), (4.3) and these are shown in Fig. 2. The theorem holds in these cases. We assume therefore that $n>4$ and that the theorem has been demonstrated for all smaller values of $n$. The graph $\Gamma_{1}$ is then a tree with $n_{1}$ vertices, where $n_{1} \geqq 2$, so it has a free vertex which is the end-point of a single red edge.

In this and the next section it is useful to have a name for vertices which are not endpoints of any red edge in $\Gamma$ : we shall call these uncoupled vertices. Those which are not vertices of a blue edge must be vertices of a single red edge, and are therefore free 
n

1

2

3

4

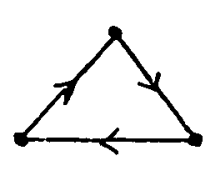

$$
\Gamma
$$$$
\text { - }
$$

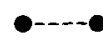

$\bullet-\cdots$

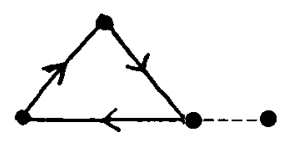

$$
\Gamma_{1}
$$$$
\bullet
$$

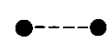

id $\tau$

id

id

FIGURE 2 Graphs of degree 1,2,3,4, which collapse to a tree.

vertices, so no ad hoc term is needed. Since $\Gamma$ is connected no vertex can be both uncoupled and free except in the trivial case $n=1$.

Returning to our inductive proof, the free vertex of the collapsed graph $\Gamma_{1}$ corresponds in $\Gamma$ either

Case 1 to a triangle $v_{1} v_{2} v_{3}$ with $v_{1}$ joined by a red edge to a vertex $w$ in $\Gamma$ while $v_{2}$ and $v_{3}$ are uncoupled; or

Case 2 to a free vertex $v_{1}$ joined to a vertex $w$ of $\Gamma$ by a red edge.

Let $\Gamma^{*}$ denote the graph obtained from $\Gamma$ by deleting, in Case 1 , the triangle $v_{1} v_{2} v_{3}$ and the red edge $w v_{1}$; in Case 2, $v_{1}$ and the red edge $v_{1} w$. Then $\Gamma^{*}$ satisfies (4.2), (4.3) and we have a pair of permutations $\left(\mu^{*}, \tau^{*}\right)$ of its vertex set. The corresponding collapsed graph $\left(\Gamma^{*}\right)_{1}$ is obtained from $\Gamma_{1}$ by deleting a single free vertex and the edge incident with it, so it is again a tree. By the induction hypothesis, $\mu^{*} \circ \tau^{*}$ is a single cycle which can be written $\left(A w_{1} w\right)$, where $A$ is a sequence giving all the vertices of $\Gamma^{*}$, except $w_{1}, w$, in some order, and $w_{1}=\tau^{-1}(w)=\tau^{*-1}(w)$. We then have the two cases illustrated in Fig. 3.

If we write $\sigma=\mu \circ \tau$ and $\sigma^{*}=\mu^{*} \circ \tau^{*}$, then each of the vertices which occur in the sequence $A$ above has the same image under $\sigma$ and under $\sigma^{*}$, so the sequence $A w_{1}$ occurs in the cycle representation of $\sigma$. By direct study of Fig. 3, we find, in Case 1,

$$
\sigma\left(w_{1}\right)=v_{1}, \sigma\left(v_{1}\right)=v_{2}, \sigma\left(v_{2}\right)=v_{3}, \sigma\left(v_{3}\right)=w, \sigma(w)=\sigma^{*}(w), \sigma=\left(A w_{1} v_{1} v_{2} v_{3} w\right)
$$

and in Case 2,

$$
\sigma\left(w_{1}\right)=v_{1}, \sigma\left(v_{1}\right)=w, \sigma(w)=\sigma^{*}(w), \sigma=\left(A w_{1} v_{1} w\right) .
$$

In both cases $\mu \circ \tau$ is a single cycle, and the theorem is proved by induction. 


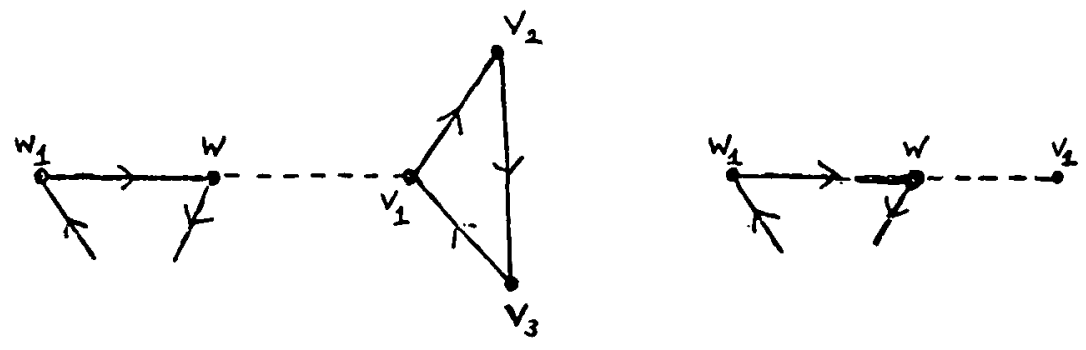

FIGURE 3

Theorem 4 immediately yields $(2,3)_{n}$-pairs for every $n$. For future use we describe the standard $(2,3)_{n}$-pair. Its graph is a sequence of similarly placed blue triangles each directed clockwise, with the right-hand base vertex of each triangle joined by a red edge to the left-hand base vertex of the next, and with one free vertex joined to the left base vertex of the first triangle if $n \equiv 1(\bmod 3)$, two free vertices joined by red edges to the left base vertex of the first triangle and the right base vertex of the last triangle if $n \equiv 2(\bmod 3)$. To be quite specific, the involutions $\mu$ are given below:

$$
\begin{array}{ll}
n=3 m & \mu=(n, 3)(n-1,5) \ldots(2 m+2,2 m-1) \\
n=3 m+1 & \mu=(1,2)(n, 4) \ldots(2 m+3,2 m) \\
n=3 m+2 & \mu=(1,2)(n, 4) \ldots(2 m+3,2 m+2) .
\end{array}
$$

In each case we can compute $\tau$ from the relation $\tau=\mu \circ \sigma$. See Fig. 4.

$n=3 m$

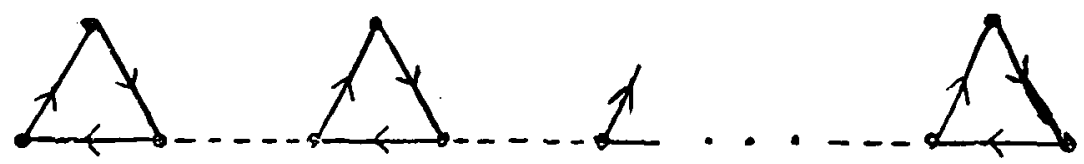

$n=3 m+1$

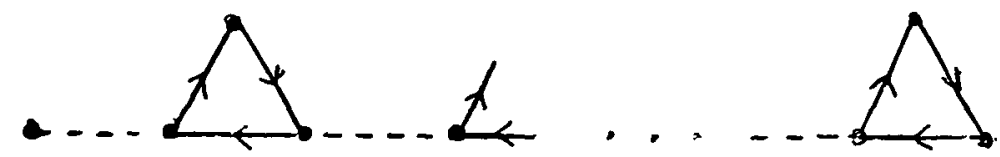

$n=3 n+2$
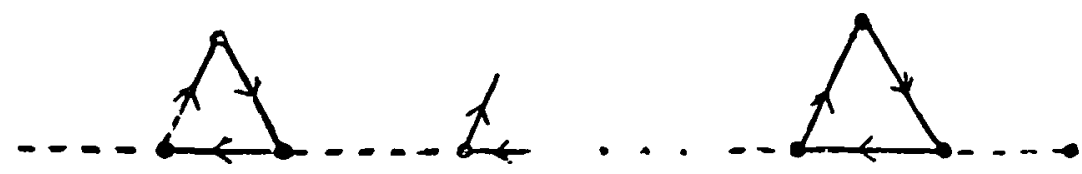

FIGURE 4 The standard graphs. 


\section{Superimprimitive $(2,3)_{n}$-pairs of prime power degree}

In this section we construct superimprimitive $(2,3)_{n}$-pairs when $n$ is a power of an odd prime. Such pairs cannot of course be regular, since the degree is not divisible by 6 , but Theorem 3 will enable us to use them as building blocks to construct regular pairs. There is no superimprimitive pair, regular or otherwise, if $n$ is divisible by 4 , because there is no superimprimitive $(2,3)_{4}$-pair.

The prime 5 will need to be treated separately, because there is only one uncoupled vertex in the graph of the unique $(2,3)_{5}$-pair. For all odd primes other than 5 , the standard $(2,3)_{p}$-pair has at least two uncoupled vertices; and for $p=3$, the graph, consisting of a single blue triangle, has no free vertex. This proves Case $m=1$ of the following theorem:

Theorem 5. If $p$ is an odd prime different from 5, there is a superimprimitive $(2,3)_{p^{m}}$ pair whose graph has at least two uncoupled vertices and such that its collapsed graph is a tree. Moreover, if $p=3$, the permutation $\tau$ is regular (or, what is the same thing) the graph has no free vertex.

Proof. Make the induction assumption that we have a $(2,3)_{p^{m}}$-pair $\left(\mu_{m}, \tau_{m}\right)$ whose graph $\Gamma_{m}$ has two uncoupled vertices, $v, w$, say, and with collapsed graph $\left(\Gamma_{m}\right)_{1}$ a tree and such that $\left(\mu_{m}, \tau_{m}\right)$ is superimprimitive.

The graph $\Gamma_{m+1}$ is then constructed by adjoining $p-1$ red edges to the disconnected graph which is the disjoint union of $p$ copies of $\Gamma_{m}^{(i)}, i=1, \ldots, p$ of $\Gamma_{m}$. For each $i$ and for each vertex $x$ of $\Gamma_{m}$ let $x^{(i)}$ denote the vertex of $\Gamma_{m}^{(i)}$ corresponding to $x$. Define the map $\phi$ from the set $V_{m+1}$ of vertices of this union to $V_{m}$, the set of vertices of $\Gamma_{m}$, by $\phi\left(x^{(i)}\right)=x$.

The graph $\Gamma_{m}$ is obtained by adding red edges

$$
\begin{aligned}
& \left(v^{(1)}, v^{(2)}\right),\left(v^{(3)}, v^{(4)}\right), \ldots,\left(v^{(p-2)}, v^{(p-1)}\right) \\
& \left(w^{(2)}, w^{(3)}\right),\left(w^{(4)}, w^{(5)}\right), \ldots,\left(w^{(p-1)}, w^{(p)}\right)
\end{aligned}
$$

to the union $\Gamma_{m}^{(1)} \cup \cdots \cup \Gamma_{m}^{(p)} . \Gamma_{m+1}$ and therefore $\left(\Gamma_{m+1}\right)_{1}$ is connected. To prove that $\left(\Gamma_{m+1}\right)_{1}$ is a tree we verify that the number of vertices exceeds the number of edges by 1 (see [8, p. 45, Theorem 9A (iii)]). Each $\left(\Gamma_{m}^{(i)}\right)_{1}$ is a tree. In their union the total number of vertices exceeds the number of edges by $p$. Adding $p-1$ edges reduces the deficit to 1 and $\left(\Gamma_{m+1}\right)_{1}$ is a tree.

Next we show that $\left(\mu_{m+1}, \tau_{m+1}\right)$ is superimprimitive. Since each red edge in $\Gamma_{m+1}$ joins a vertex $x^{(i)}$ to either $\left(\mu_{m}(x)\right)^{(i)}$ or $\left(\mu_{m}(x)\right)^{(i \pm 1)}$ if $x$ is $v$ or $w$; and since each blue edge comes from a blue edge in one of the $\Gamma_{m}^{(i)}$, so that

$$
\tau_{m+1}\left(x^{(i)}\right)=\tau_{m}(x)^{(i)}
$$

we deduce:

The sets $\left\{\phi^{-1}(x) \mid x \in V_{m}\right\}$ form a block system compatible with the permutations $\mu_{m+1}, \tau_{m+1}$. These blocks are in one-to-one correspondence with $V_{m}$ and the permutations $\mu_{m+1}, \tau_{m+1}$ induce the permutations $\mu_{m}, \tau_{m}$ of $V_{m}$. 
Since $\left(\mu_{m}, \tau_{m}\right)$ is superimprimitive, it follows that, for $1 \leqq r \leqq m$ it has a system of $p^{r}$ blocks $\left\{B_{1}, \ldots, B_{p r}\right\}$ with which it is compatible. Then $\left\{\phi^{-1}\left(B_{1}\right), \ldots, \phi^{-1}\left(B_{p r}\right)\right\}$ is a block system compatible with $\left(\mu_{m+1}, \tau_{m+1}\right)$, which is therefore superimprimitive.

If $p=3$, none of the $\Gamma_{m}^{(i)}$ has a free vertex, so, since only edges and no vertices are added to make $\Gamma_{m+1}$, it follows that $\Gamma_{m+1}$ has no free vertex.

Finally, $\Gamma_{m+1}$ has the uncoupled vertices $w^{(1)}, v^{(p)}$ and the proof of Theorem 5 by induction is complete.

Theorem 6. There is a superimprimitive $(2,3)_{5 m}$-pair whose graph $\Gamma_{m}$ has at least one uncoupled vertex and at least one free vertex, and such that its collapsed graph is a tree.

Proof. We give only the inductive construction of the graph which yields the desired pair. The proof that it has the properties claimed is similar to the proof of Theorem 5 and is omitted. Suppose then that $\Gamma_{m}$ has been constructed, that $v$ is a free vertex and that $w$ is an uncoupled vertex. Then $\Gamma_{m+1}$ is obtained by adding to the disjoint union of five copies $\Gamma_{m}^{(1)} \cup \Gamma_{m}^{(2)} \cup \cdots \cup \Gamma_{m}^{(5)}$ of $\Gamma_{m}$ two new red edges $\left(w^{(1)}, w^{(2)}\right),\left(w^{(4)}, w^{(5)}\right)$ and a new blue triangle $\left(v^{(2)}, v^{(3)}, v^{(4)}\right)$. $\Gamma_{m+1}$ then has the uncoupled vertex $w^{(3)}$ and the two free vertices $v^{(1)}, v^{(5)}$. The induction is started off with the standard pair $(2,3)_{5}$ described at the end of Section 4.

\section{Regular superimprimitive pairs}

Let $n=6(2 g-1)$, where $n$ has the prime factorization

$$
2.3^{\alpha_{1}} p_{2}^{\alpha_{2}} \ldots p_{k}^{\alpha_{k}}
$$

where $\alpha_{1} \geqq 1$ and $5 \leqq p_{2}<p_{3}<\cdots<p_{k}$ are the prime factors, other than 2,3 , of $2 g-1$.

Let $\left(\mu_{0}, \tau_{0}\right)=((12), i d)$ of degree 2 , let $\left(\mu_{1}, \tau_{1}\right)$ be a superimprimitive $(2,3)_{3}{ }^{\alpha_{1}}$-pair with $\tau_{1}$ regular, and for $r=2, \ldots, k$, let $\left(\mu_{r}, \tau_{r}\right)$ be superimprimitive $(2,3)_{p_{r}} a_{r}$-pair. Finally, let

$$
\mu=\mu_{0} \times \mu_{1} \times \cdots \times \mu_{k}
$$

and

$$
\tau=\tau_{0} \times \tau_{1} \times \cdots \times \tau_{k}
$$

Then, by Theorem $3,(\mu, \tau)$ is a regular superimprimitive pair.

Thus regular superimprimitive pairs exist for every value of $g$, as Hussein surmised. Even using our construction, however, which certainly does not yield all possible pairs, we can see that the superimprimitive pair is by no means unique. Thus if we begin with a prime $p$ and the standard graph has more than two uncoupled vertices, we have a choice of different pairs of vertices $v, w$ to start off the induction. Also there is no reason why we should not use new blue triangles in the building process (as was done for $p=5$ ) whenever $p$ is a prime larger than 7 and congruent to $2 \bmod 3$. In addition to this, if the degree is not very small, we have a choice of many possible trees for the graph $\Gamma_{1}$ other than the rather dull one associated with the standard graph. In addition to the 
uniqueness noted by Hussein for $g=1,2,3$, there is only one regular superimprimitive pair for $g=4$. For $g=6$, however, uniqueness no longer holds. To see this, note that, in addition to the standard graph of degree 11, we have the two distinct graphs shown in Fig. 5. There are also more than one regular superimprimitive graphs for $g=5$, but it is slightly more difficult to illustrate these, since the prime power constituent $27=3^{3}$ occurs.

All but one of the graphs with 11 vertices give pairs $(\mu, \tau)$ which generate $A_{11}$. The remaining one is the first graph shown in Fig. 5 which generates the exceptional permutation representation of $\operatorname{PSL}(2,11)$ (see [9, p. 286, Theorem 262]). The Mathieu group $M_{11}$ does not appear. These facts have been verified using the Cayley program on the University of Pittsburgh VAX/VMS. Our thanks to Dr John Cannon for making the software available and to John Burkhardt for getting it established on the computer, and for his patience in correcting my first stumbling attempts to use it.

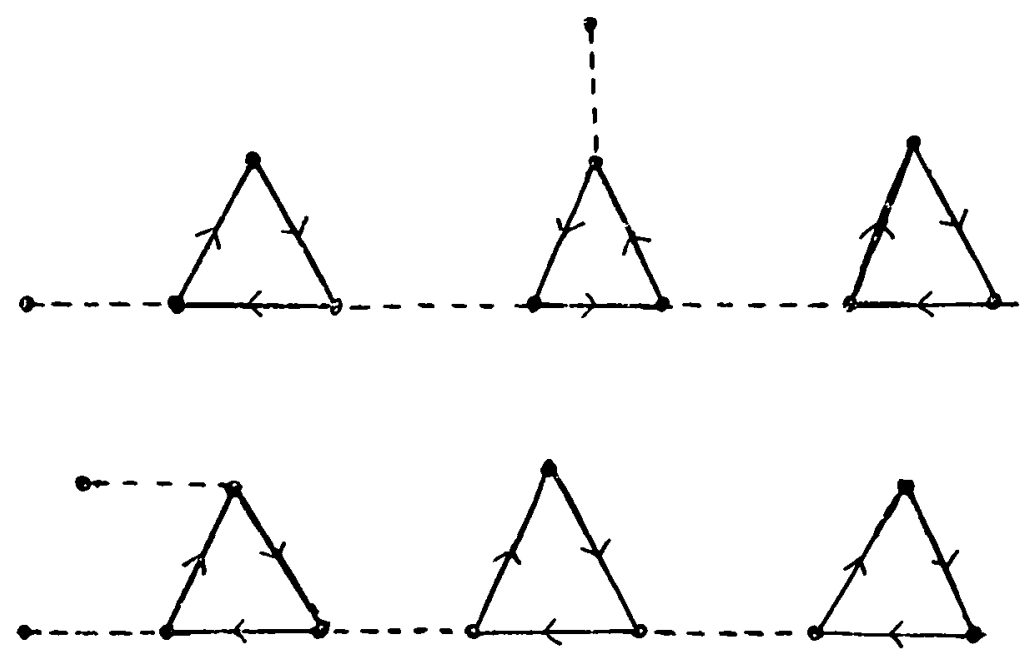

FIGURE 5 Two distinct graphs, other than the standard graph, of degree 11.

Note added in proof. Since writing this paper, I have been shocked and saddened by the news of Hussein's sudden death. The concept (and the name) "superimprimitive" is his invention, and I hope this paper helps it to live on.

\section{REFERENCES}

1. M. D. E. CONDER, Generators for the alternating and symmetric groups, J. London Math. Soc. 22 (1980), 75-86.

2. M. D. E. Condor, More on generators for the alternating and symmetric groups, Quart. J. Math., Oxford (2) (1981), 137-163.

3. Marshall Hall, The Theory of Groups (Macmillan, New York, 1961).

4. A. A. Hussein Omar, On some permutation representations of $(2,3, n)$-groups (Ph.D. Thesis, University of Birmingham, England, 1979). 
5. A. M. MACbeath, Generic Dirichlet polygons and the modular group, Glasgow Math. J. 27 (1985), 129-141.

6. W. W. Stothers, Subgroups of infinite index in the modular group, Glasgow Math. J. 19 (1978), 33-43.

7. W. W. Stothers, The number of subgroups of given index in the modular group, Proc. Roy. Soc. Edinburgh 78A (1977), 105-112.

8. R. WiLSON, Introduction to Graph Theory (Longman, London, 1975).

9. L. E. Dickson, Linear Groups, with an exposition of the Galois field theory (Dover reprint, New York, 1958).

Department of Mathematics and Statistics

UNIVERSITY OF PITTSBURGH

PitTSBURGh, PA 15260 , U.S.A. 